www.itcon.org - Journal of Information Technology in Construction - ISSN 1874-4753

\title{
INTEGRATION OF BUILDING SERVICE SYSTEMS IN ARCHITECTURAL DESIGN
}

SUBMITTED: January 2019

REVISED: May 2019

PUBLISHED: February 2020 at https://www.itcon.org/2020/7

EDITOR: Turk Ž.

DOI: $10.36680 /$ j.itcon. 2020.007

\author{
Wael Abdelhameed, Associate Professor, \\ Applied Science University, Bahrain; \\ wael.abdelhameed@fulbrightmail.org \\ Weldy Saputra, Lecturer, \\ University of Bahrain, Bahrain; \\ wsaputra@uob.edu.bh
}

\begin{abstract}
The paper investigates the importance of building service systems, BSS particularly how they should be taken into consideration during the early architectural design phases. The integration of those systems inside the building in the early phases of design will save cost and prevent time-consuming modifications. Due to the late integration of the building service systems BSS in the design, negative impact on both the exterior and the interior, may occur. Within the building industry, there has been increasing interest to the building service systems BSS integration, in order to enhance design outcomes, and to detect or even avoid the service systems' clashes and conflicts. An academic-course projects are used to highlight the importance of $3 D$ digital modelling in disclosing possible clashes and conflicts between the building service systems BSS, particularly the plumbing systems in one hand, and between the design itself and those systems on the other hand. A literature review to cover methods used to coordinate all service systems is conducted. Furthermore, from the construction industry more data are collected and analysed to add the practical perspective. Not only some coordination cases from regional construction projects but also researches focused upon construction industry, are employed to shade more light on the benefits of the integration of the building service systems BSS in the conceptual phases of architectural design.
\end{abstract}

KEYWORDS: building service systems, MEP, conceptual design phases, architectural education

REFERENCE: Wael Abdelhameed, Weldy Saputra (2020). Integration of building service systems in architectural design. Journal of Information Technology in Construction (ITcon), Vol. 25, pg. 109-122, DOI: 10.36680/j.itcon.2020.007

COPYRIGHT: () 2020 The author(s). This is an open access article distributed under the terms of the Creative Commons Attribution 4.0 International (https://creativecommons.org/licenses/by/4.0/), which permits unrestricted use, distribution, and reproduction in any medium, provided the original work is properly cited. 


\section{INTRODUCTION}

Building service systems, BSS are essential elements in the design process, which in most cases has great impacts on architectural and structural designs. The BSS are piping network of fresh water and wastewater -sewage-, electrical installation network, air conditioning systems, fire prevention and protection systems, and communication systems - external and internal-, which can be summarised as MEP: Mechanical, Electrical and Plumbing. Including the service systems and their components into the architectural and structural designs, and the coordination between these systems help avoid major obstacles before the construction process.

The MEP design and coordination have importance role in the design process. The coordination processes used to be carried out by overlaying the two-dimensional drawings of different service systems, each of which is designed by different specialised designers. The processes basically depended upon the experiences of architects and structural engineers to avoid the possible conflicts and to include the systems' components and spatial requirements into the design and its spaces. Errors might not be fully detected by these traditional processes till the construction stages. Identifying conflicts in the 2D-drawings of service systems used to be an unsuccessful process, due to two main reasons: depending upon designers' experiences by delaying the systems coordination to later design stages and using 2D methods and 2D drawings in detecting the errors and conflicts. These possible errors or conflicts negatively affect the projects in many aspects, particularly in case of being undetected after the construction completion which consequentially impact the project's spaces to accommodate the systems' components and requirements.

In the current design applications, architects attempt to include the spatial requirements of the service systems and their components in the design process as early as possible. The benefits resulted from this integration of BSS in the early design processes remarkably save time, effort, and budget.

Using 3D digital modelling in the processes of design and coordination not only improves the designers' raw imagination by representing a 3D model including the components of the service systems, but also eliminates the errors generated from the lack of designers' experiences by visually presenting all systems' components even in case of not using one model for all service systems. Employing digital modelling eases the processes of coordination and design, and makes them more accurate. Authorities, stakeholders and decision makers will gain many advantages, such as: creating a detailed model of both the design and the service systems which makes their decisions more reliable and accurate.

BIM is an approach and a process in which the design model potentially includes various building information of different components and spaces, in order for the users to visualise, manage, analyse and/or design in a better way. BIM approach offers an effective assistance represented in making a multidisciplinary model that has BSS in one detailed model, which helps discover and solve any obstacles of overlapping or/and conflicting. Unlike other digital tools that help the imagination capabilities of architects or architecture students, BIM proceeds beyond to unveil and expose possible problems that may appear in the later processes of designing and construction.

Both the architecture students in the academia level and the graduates in the industry market, lack the technical knowledge required into the architectural design. According to two surveys of RIBA -Royal Institute of British Architects- and ACENZ -Association of Consulting Engineers New Zealand- in 2007, graduates do not have the design knowledge to solve the technical/construction details inside the designed spaces (Abdelhameed, 2018). The two studies highlighted that the graduates moreover lack the knowledge to build what they design (Abdelhameed and Saputra, 2019).

\section{GOAL AND OBJECTIVES}

The main goal of this study is to present a method of applying the technical knowledge of BSS in the early phases of architectural design, through an academic course, and proving its effectiveness through quantitative and qualitative analysis. The paper aims at achieving this goal through the following objectives: a) exploring the benefits to integrate the building service systems into the conceptual design processes; b) investigating the 3D digital modelling in an academic course of BSS where the students make detailed requirements of one service system in their designs; $c$ ) focusing on the details of piping network of fresh water and waste water particularly in the primary data of the students' designs, rather the other BSS; and finally d) using some practical cases from the regional construction projects to include more focus upon the advantages of the BSS integration into the conceptual design stages. 


\section{METHODOLOGY}

In terms of achieving the paper's objectives, and highlighting their benefits and applicability, the researchers apply the following methodology that is divided into three-folded tools:

- Use academic-course projects, to highlight the importance of 3D digital modelling to disclose possible clashes and conflicts between the building service systems particularly the plumbing systems in one hand, and between the design itself and those systems on the other hand.

Within this methodological tool, a questionnaire is conducted to measure the academic benefits and the student opinions. The quantitative analysis of the student replies, as well as the qualitative analysis, helps investigate and achieve the paper's objectives.

- $\quad$ Another methodological tool: analysis of the qualitative data from the related researches, is applied to cover all BSS. The primary data from the presented course, related only to the plumbing systems, are not enough to cover the whole paper scope.

- The third tool is to utilise not only some service-systems coordination cases from projects of a regional construction firm but also researches from the literature review focused upon the construction industry, to shade more light on the benefits of the integration of all building service systems in the conceptual phases of architectural design.

The collected data of this methodological tool from the regional construction projects, covering all building service systems, focus upon the execution stages and the final design drawings.

The first reason to use multiple methodological tools is to achieve all the paper's objectives in a method that comprehensively combines the educational process of academia and the practical projects of industry. Another reason is that to cover each objective of the paper by more than one methodological tool and one type of analysis.

\section{LITERATURE REVIEW}

Representing an opposite point of view from this paper, Matsubayashi and Watanabe (2015) argued that it can be difficult to grasp the relationships between various MEP elements when they are modelled in a visually complex, three-dimensional manner. They maintained that the relationships between the elements of these systems can be easily understood when using traditional schematic diagrams, generated from BIM (Matsubayashi and Watanabe, 2015).

The research work, however, related to the paper's subject offers many researches and practical case studies that highlight not only the importance of 3D modelling into the processes of designing the service systems, but also the beneficial use of BIM that is represented into conflict detection through the multidisciplinary model. What BIM offers to the MEP coordination is indispensable and goes beyond mere modelling, yet the paper's literature review attempts to cover the $3 \mathrm{D}$ digital modelling method as a main tool of designing and coordinating the building service systems. There are not enough researches in the area of plumbing systems; only a few numbers of researches have been identified.

The literature review, moreover, is related to the two objectives of the paper: building service systems' coordination, and the use of 3D digital modelling tool, which can be classified into the following points: courses in academia; plumbing systems; MEP systems design and coordination; and 3D modelling tool and BIM.

\section{Courses in Academia}

Palomera-Arias and Liu (2015) at the University of Texas at San Antonio, presented the results of student surveys that were used specifically to assess the effectiveness of the BIM exercise in presenting the MEP topics. The surveys' results highlight the positive impact on MEP systems design and coordination. They discussed the course details such as: the course organisation, the development of the MEP Systems course laboratory exercises, and the specific topics covered (Palomera-Arias and Liu, 2015).

Gegana and Widjarnarso (2015) at University of Indonesia and Institute of Technology Bandung, introduced the fundamental student learning outcomes that has been reached in 2 BIM courses in both universities. Samples of students' work have been presented and discussed. They stated that the emerging globalization brings us the 
awareness about the need to incorporate BIM into architecture school curriculum in order to replace the conventional CAD documentation (Gegana and Widjarnarso, 2015), used to be the main tool for MEP systems' design and coordination.

Stone and King (2015) at Woodbury University Bachelor, presented a Detail Design course of the Interior Architecture programme, in which students were encouraged to use BIM to look at the issues surrounding sustainable design through an investigation into the theme of design for disassembly. They explained that the disassembly method facilitates the systematic study of geometrical relationships between component elements and their material qualities (Stone and King, 2015).

\section{Plumbing Systems}

The use of construction knowledge and planning experience of design, engineering, construction and supply for design optimization is defined as constructability (Othman, 2011). An innovative framework to facilitate the integration of construction knowledge and contractor's experience in the design process is developed (Othman, 2011), that can be used to improve the building service systems including the plumbing systems.

Dantas Filho et al. (2015) present an application of virtual design and construction, VDC, that improves the plumbing system design, and maintain that Request For Information, RFI are financially valuable to the constructor. They recommended to identify the RFI types in plumbing system design process or during virtual construction to improve conception, production and managing processes of new water/sanitary facility designs (Dantas Filho et al., 2015).

Cao et al. (2018) investigated the shortest connecting plumbing path between hydraulic components in the compact design, and proposed a visibility graph that can be expanded to 3D applications. They developed a technique using 3D modelling to address the inefficiency issue and made a comparison to the traditional techniques (Cao et al., 2018).

Arugam et al. (2018) presented a home scale greywater treatment system that is a combination of three water treatments: physical, chemical, and biological treatment. In the methodology they used, the system design was modelled into a 3D prototype that is used to evaluate the system design performance. The final design is represented in 3D model with all the mechanical parts.

Hassanain et al. (2018) proposed 36 factors divided into six categories: planning phase of the project; design of mechanical, electrical and plumbing MEP systems; construction of MEP systems; operation and maintenance of MEP systems; owner; and design team and tools used, which impact building service systems coordination in general.

\section{MEP Systems Design and Coordination}

Many researchers described MEP coordination as the most challenging task, which requires iterative and experience-driven and entails considerable time and human resources (Wang and Leite, 2016; Ashuri et al., 2014), as the process is manual, error-prone, time-consuming, and expensive. There is no a systematic way to create lessons learned in terms of supporting future decision making, and introduced schema that can be used to capture clash features and associated solutions during MEP coordination (Wang and Leite, 2016). Akponeware and Adamu, (2017) stated that a search conducted within peer reviewed databases, as well as Internet, did not reveal any specific tool designed to automatically assist professionals achieve clash avoidance.

After completing the designs, the coordination process begins by having several meetings, in which the 2D drawings of different MEP systems, developed by engineers, are sequentially compared and overlaid on a light table to detect spatial conflicts and clashes (Ashuri et al. 2014). The coordination process might also result in the structural and architectural design modifications.

The code logic applied in the algorithms of clash detection is to determine the proximity of two or more objects and whether their geometries collide in virtual space. Van den Helm et al. (2010) categorized clash detection algorithms into: a) comparing shapes, b) comparing axis aligned bounding boxes, c) the Ray triangle intersection, and d) the industry foundation classes -IFC- structure method. The process has been further developed to include more categories, namely: hard clashes, soft clashes and time clashes (Guangbin et al., 2011). Akponeware and Adamu, (2017) highlighted the increased marketing of clash detection software, whose success at finding clashes are touted by their developers and users. 
BIM potential to detect clashes and conflicts may not be fully effective, yet it eliminates most of these coordination problems. Wang et al. (2016) presented a case study, whose results have the following: $78 \%$ of the collisions are ineffective when using the BIM tools for collision detection. In the same time, a significant number of effective collisions -102 collisions- are still manually detected by the designers and contractors.

In another perspective, Benning et al. (2010) argued that the most effective way to avoid clashes between all service systems is to ensure clash avoidance, through more direct communication between designers to resolve clashes in case of occurrence.

Shen et al. (2009) presented a critical review of the state-of-the-art systems integration and collaboration technologies, standards and tools in the architecture, engineering, construction, and facility management AEC/FM industry. They included data modelling and integration in general as the interoperability that is part of the Information Technology integration and collaboration in the industry (Shen et al. 2009). Building service systems are viewed as part of data modelling.

Akponeware and Adamu (2017) highlighted the reasons of clash occurrences in the MEP systems, as follows: a) isolated working was the prime cause of high occurrences of clashes; b) the professional qualifications of design practitioners, and non BIM specific training in case of using BIM; and C) the use of cloud-based common data environments, CDEs does not facilitate clash avoidance in the early design stage where designers of multidisciplinary backgrounds work on different models. They concluded that the engagement in concurrent cocreation and one multidisciplinary model, through a transparent and inclusive process could be the solution of MEP systems' coordination (Akponeware and Adamu, 2017).

Farooq et al. (2017) investigated with supportive case studies, the potential applications of BIM in electrical system design and analysis, and concluded that seamless integration of semantic information system of BIM with Geographical Information System, GIS can be very useful for electrical grid optimization and city energy modelling.

Korman et al. (2010) recommended the following order in MEP coordination: HVAC air ductworks, sanitary drainage system, HVAC process piping, fire protection piping, water distribution piping, electrical. They highlighted that the structure should not been changed unless absolutely inevitable, and proceeded to set the MEP coordination to start after the integration of architectural and structural models. The location of major plant and equipment is confirmed first, after that HVAC system comes second, and the others come later (Korman et al., 2010).

Pérez-Lombard et al. (2011) classified the HVAC prescriptive requirements into six categories: equipment minimum efficiencies, fluid distribution systems, HVAC control, ventilation, heat recovery, and free-cooling. They maintain that these categories are responsible to reduce energy consumption of the HVAC system in buildings (Pérez-Lombard et al., 2011), which in turn is a unique benefit of designing and planning all service systems.

\section{D Modelling Tool and BIM}

Akponeware and Adamu, (2017) stated that based on different studies, the introduction of digital systems improves coordination, collaboration and communication among the sundry design disciplines involved in a project. 3D digital modelling is one of these systems used in the project delivery.

Staub-French and Khanzode (2007) suggested a process for 3D design coordination, based on US procurement practice. The process employs a 3D modelling tool through the BIM use. Within the suggested 10 steps of the process, three steps, namely: integrate discipline-specific 3D models; identify conflicts between components/systems; and at the end develop solutions for the conflicts identified, can be conducted easily in case of using BIM.

Three knowledge domains are required for MEP coordination, namely, design, construction, and operations and maintenance (Korman et al. 2003). ITcon published a special issue: Case Studies of BIM Use, to show the benefits and challenges of using building information modelling for stakeholders in the building process (Olofsson et al., 2008).

Wang et al. (2016) offered a practical BIM framework that integrates the MEP layout from preliminary design to construction stage. They propose five levels of details of BIM models in this framework, namely, 3D MEP 
preliminary design model, 3D MEP detailed design model, 3D MEP construction design model, MEP construction model, and MEP prefabrication model.

Yang et al. (2014) found in the case study analysis of China that although the use of BIM reduces the costs of manual MEP coordination, this use may not save overall design time as traditional 2D design is still dominant in China. They conclude that once BIM is more widely used in China, the 3D design tool will replace 2D design method and the whole process will be revised accordingly (Yang et al., 2014). They recommend 3D modelling to be the tool to plan and integrate the MEP systems inside the buildings using the BIM method. They argued that the 2D design stage has no benefits when 3D method is used (Yang et al., 2014).

Akponeware and Adamu (2017) identified structural and HVAC components as the main building systems involved in hard clashes. They stated that to resolve the identified clashes, designers communicate using different approaches, other than BIM approach through CDE (Akponeware and Adamu, 2017).

\section{ACADEIMC COURSE}

The BSS course objective proceeds beyond studying the impact of BSS details and components on a building as a whole design product, to investigate this research study's goal of bridging the gab in the knowledge between architectural design in one side and BSS design in the other side.

The BSS course is a course taught to the third-year students in the University of Bahrain. A prerequisite of BSS course is a Building Construction III course, in which advanced knowledge of building construction, building components, building technology and structural systems is provided.

As an introduction of the BSS course, the students study about the practice relationship as well as some misconduct practices between architectural design, structure and BSS to create a comfortable, safe and sustainable design. The course includes piping systems that apply in the building such as fresh water sources, and fresh-water filtering systems in different scales: large of a city or a town and small of house, as well as fresh-water distribution systems which are divided into two main systems, direct and indirect, including cold water and hot water. Moreover, sewage systems including drainage effluents are covered in the course. Building codes related to all building service systems are covered in the course. Other types of BSS are also included in the course such as fire protection systems, electrical systems.

The students, after learning the relationships between these systems and the architectural design as well as the structure, can design the components and the spatial occupation required by these systems into the architectural design and the structural system used. The course's project is to individually design by using 3D modelling the service systems explained in the course, into the design of the previous design studio course. No specific 3D modelling programme was required; it was up to each student's knowledge and background. Of the programmes used by the students are Revit, AutoCAD Architecture, and AutoCAD.

\section{QUESTIONNAIRE}

At the end of the course, the students of two sections, 40 students, were asked to answer a questionnaire and provide their opinions regarding the process of using 3D modelling in designing and planning the components of plumbing systems. The analysis of the questionnaire questions followed by a discussion of the questionnaire replies, are in the following part.

\subsection{Quantitative and Qualitative Analysis}

The paper presents each question analysis quantitatively and qualitatively, and then proceeds to provide comprehensive analysis to the questionnaire results.

\subsubsection{Pipe network system}

The first question was general to measure the 3D modelling use in terms of understanding the pipe network system. $87.5 \%$ of the respondents find that the use of 3D digital modelling has medium, strong, and very strong effect on how they understand the pipe network system, Fig. 1. Yet, $12.5 \%$ find that the effect ranges between weak effect and no effect at all. 


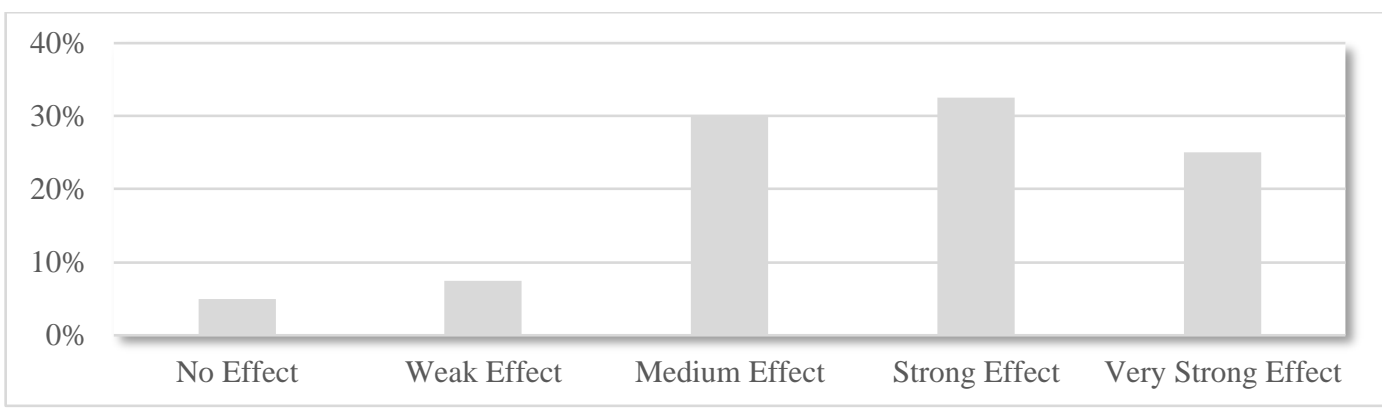

FIG. 1: Understanding the pipe network system in general.

\subsubsection{The design of network system inside the building}

The design and the capacity of pipe network systems and their critical points/connections inside the building may affect the building components, such as architectural spaces and structural system. Service systems such as Heating, ventilation, and air conditioning, HVAC can be also affected.

$82.5 \%$ of the respondents find that the 3D digital use has medium, strong, and very strong effect on how they design the capacity of the pipe network systems, and specify its connections inside the building. On the other side, $17.5 \%$ of the respondent replies are between weak effect and no effect at all, Fig. 2.

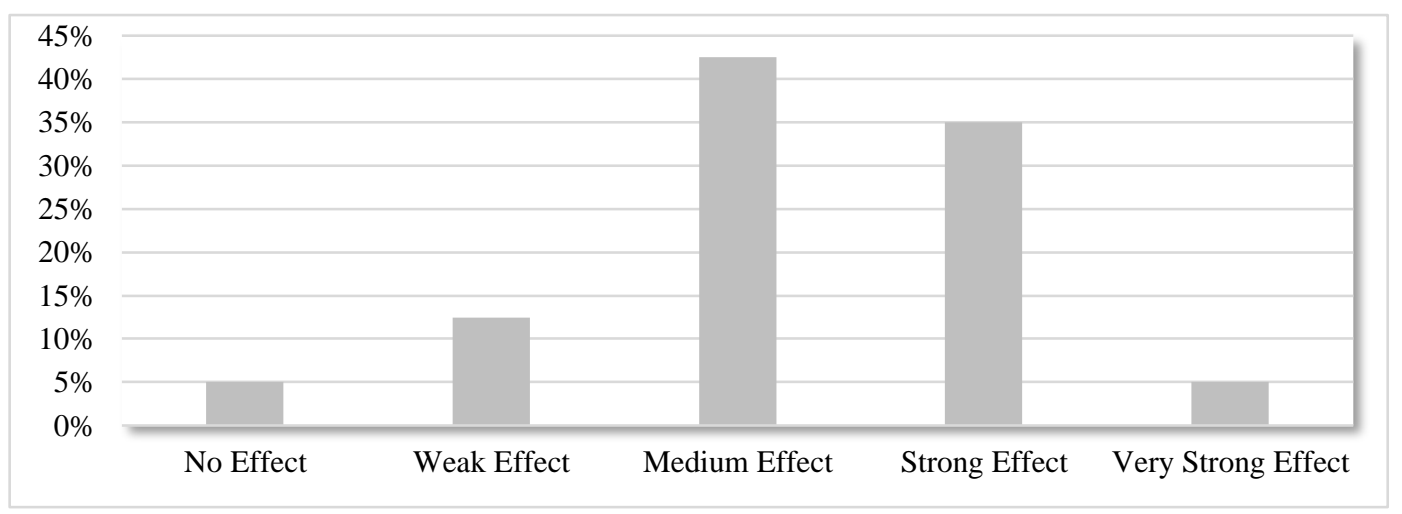

FIG. 2: 3D modelling impact on the design and the capacity of pipe network systems and their critical connections

\subsubsection{The relationship with the structural system}

Regarding the relationship between the structural system and the pipe network systems inside the building, 30\% the respondents find that 3D digital use has weak effect and no effect at all, Fig. 3, which is viewed by the researchers as a large number. On the other side, $70 \%$ of the respondent replies lay between medium, strong, and very strong effect.

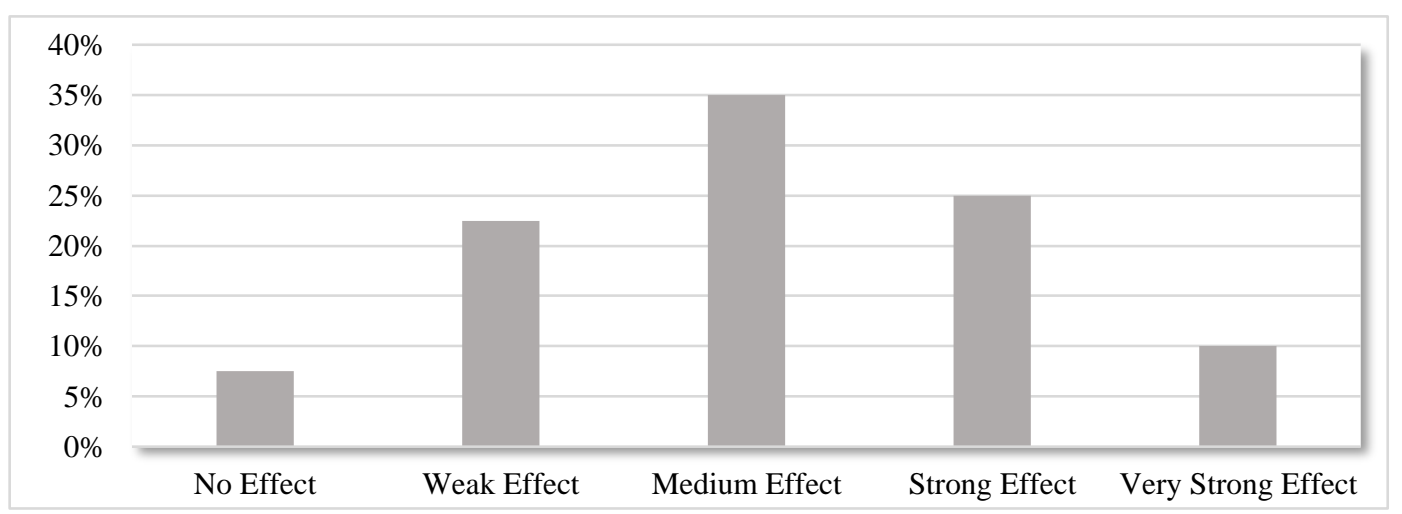

FIG. 3: 3D modelling impact on understanding the relationship between the structural system and the pipe network systems 


\subsubsection{The connection between the sanitary fixtures and the pipes}

Fig. 4 shows the replies of how 3D digital modelling affects designing and understanding the connections between sanitary fixtures and the pipes. $17.5 \%$ of the replies are between weak effect and no effect at all. On the other side, $82.5 \%$ of the respondents find that the 3D digital use has medium, strong, and very strong effect on how they design and understand the connections between sanitary fixtures and the pipes.

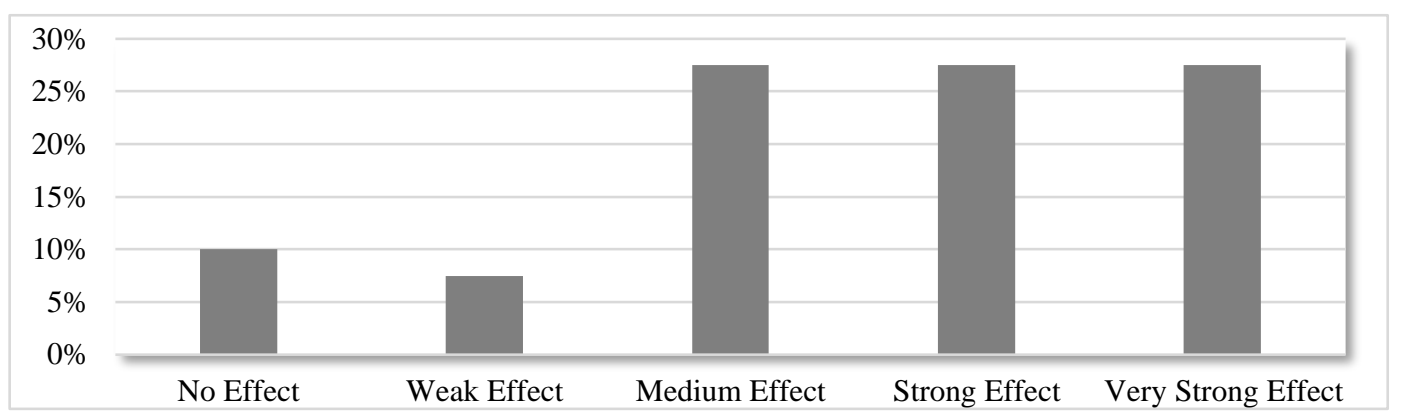

FIG. 4: 3D digital modelling impact on how to design and understand the connections between the sanitary fixtures and the pipes.

\subsubsection{Achieving an economic pipe layout design}

In the question regarding the use of 3D digital modelling to achieve an economic pipe layout design in terms of short lengths, fewer connections, etc., the student relies were varied. $32.5 \%$ of the replies are between weak effect and no effect at all. $27.5 \%$ of the replies find the modelling use has medium effect on their design, while $40 \%$ of the replies are between strong effect and very strong effect, Fig. 5.

Although the large percentage of replies supports the 3D digital modelling use in this area, the replies' variation indicates that the modelling use helps more effectively in intersections, conflicts, or clashes, rather the general design layout.

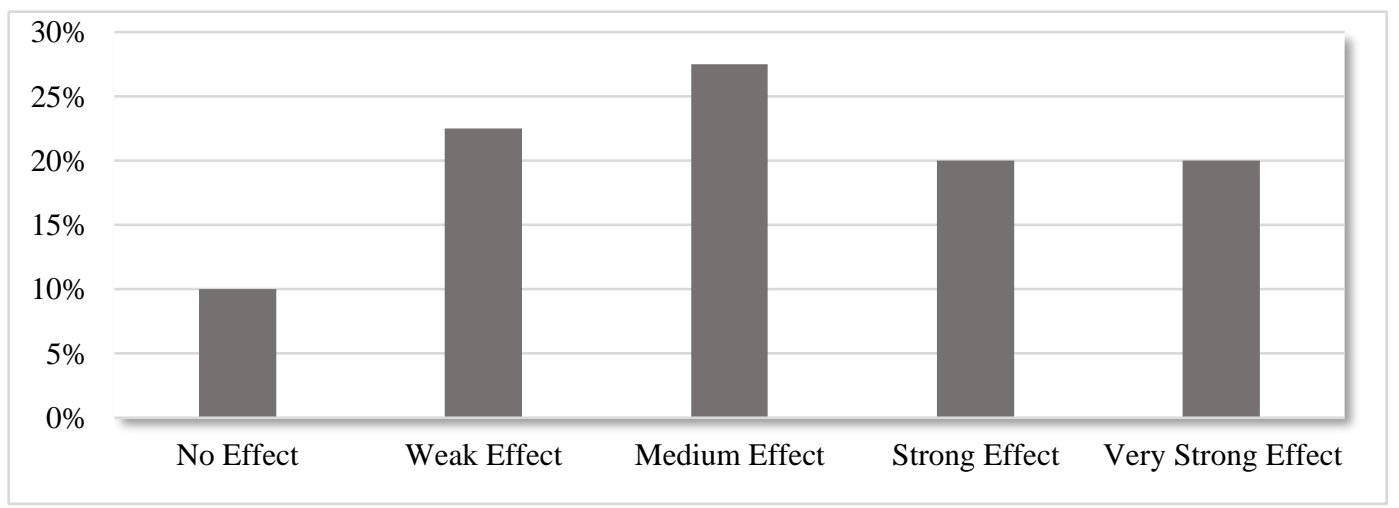

FIG. 5: 3D digital modelling to achieve an economic pipe layout design.

This question area is related to not only understanding but also imagining the pipe layouts in one side, and the structural system, other building components, and other building service systems in the other side. Both understanding and imagining processes varied from one student to another, which was reflected in their replies.

\subsubsection{The details of pipe network and their impact on the architectural form}

Fig. 6 presents the question replies of the effect of 3D digital modelling use on the details of pipe network systems and how these systems details may impact the architectural form, i.e. facades, shafts, interiors, etc. $25 \%$ of the replies are between weak effect and no effect at all. $22.5 \%$ of the replies find the modelling use has medium effect on their design, while $52.5 \%$ of the replies are between strong effect and very strong effect, Fig. 6. 


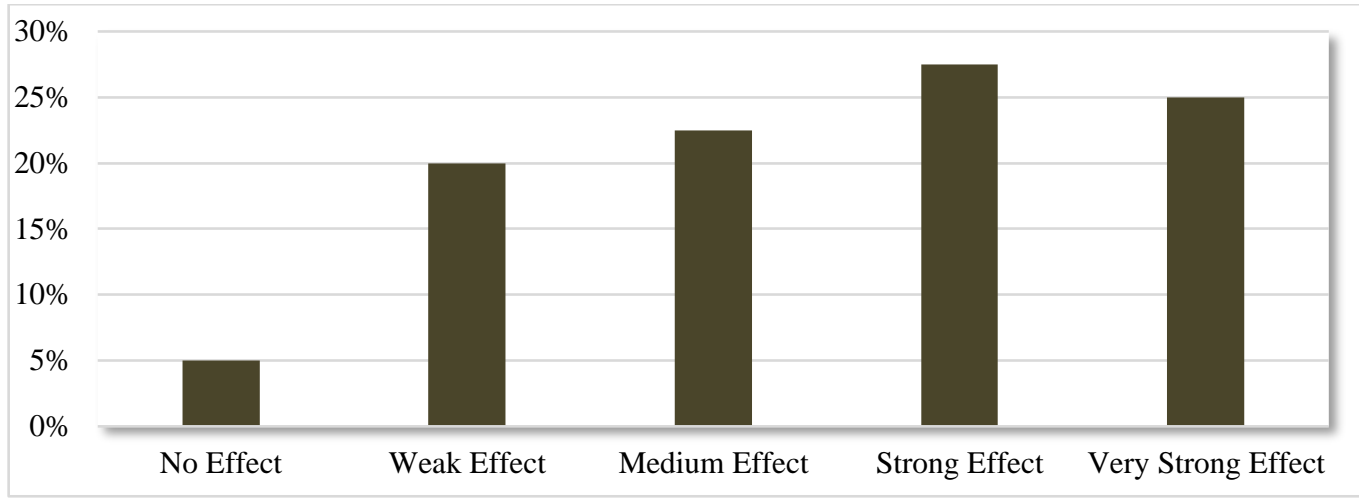

FIG. 6: 3D digital modelling use in the pipe network systems and their impact on the architectural form.

\subsection{Discussion on the Questionnaire}

The questionnaire focused upon the plumbing systems that were covered in the academic course. The processes of understanding and designing the plumbing systems and their details, as an example of one service system, include imagining skills and knowledge background of structure and other related service systems, which vary from a group of students to another. Having these factors and variables into account, the researchers did not include other service systems that are covered into a different academic level by other courses.

Fig. 7 shows all the questionnaire replies according to the number of students. In the first three questions that are related to the systems' pipe network and other building components, the effect of using 3D digital modelling demonstrates itself in a clear way. In the fourth question of the connection between the sanitary fixtures and the systems' pipes, the effect is still highly recognised.

The medium effect appears as a recognisable selection in all the questions' replies, although the no-effect appears as the least selection in all the questions' replies and the weak-effect as the second least selection in the first four questions' replies.

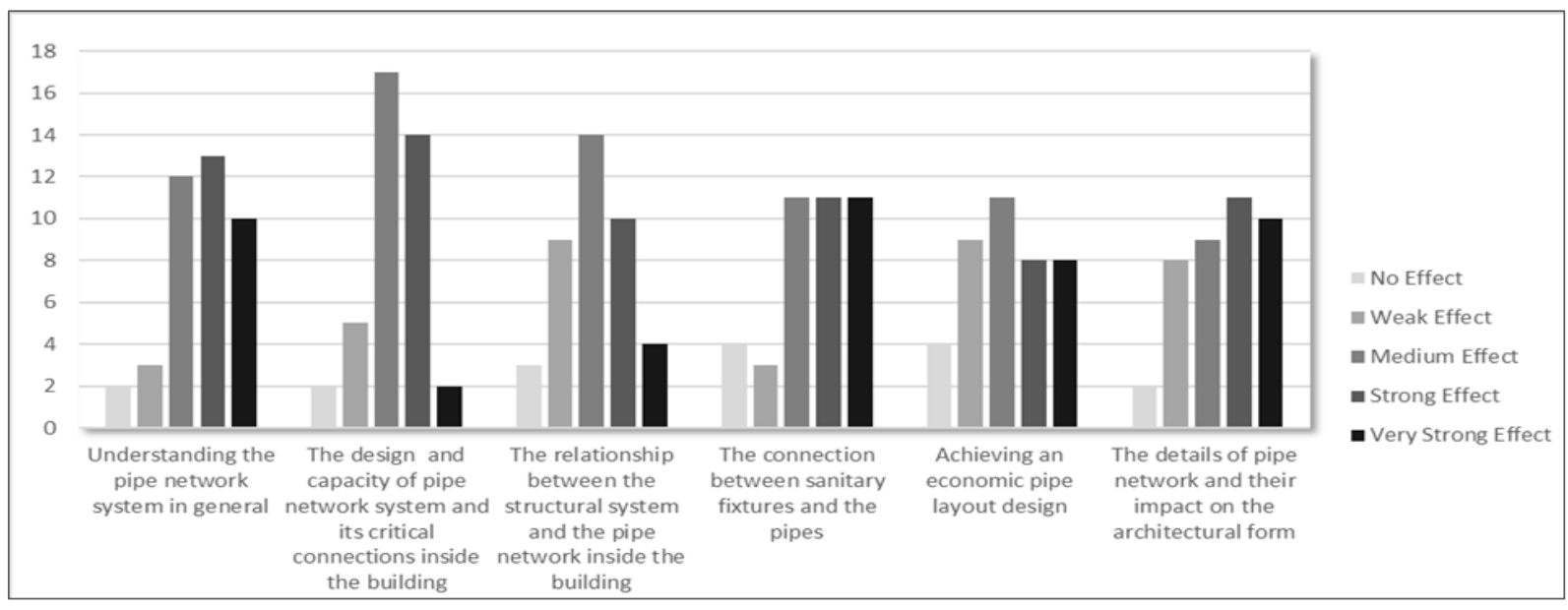

FIG. 7: Questionnaire replies.

In the last two questions regarding the economic design and the impact on architectural form, the effect of 3D digital modelling use is still with high effect although both no-effect and weak-effect are more recognised comparing to the other previous questions.

\section{PRACTICAL PROJECTS CASES}

Although the 2D drawings are still extensively used in every aspect of a building project, there is a strong movement led by the architects to transform to 3D models (Shen et al., 2010). After completing the designs, the MEP coordination process begins by holding meetings between the representatives of the general contractor and specialty trades (Ashuri et al., 2014). 
The MEP systems coordination influences the productivity of all designers of multidisciplinary backgrounds involved in the design process. Any errors or mistakes during designing or constructing the project in the MEP systems would lead to time consuming tasks, budget waste, labour time increment, and project time extension.

The next two sections cover respectively: the practical projects from different countries through a literature review, and certain project cases from a regional construction firm showing the clash coordination using 3D modelling, BIM particularly. The purpose of investigating these practical cases is to explore the lack of architectural knowledge appeared in the two surveys of Riba and ACENA in the practice level, in order to avoid this lack in the academic level.

\subsection{Global examples from literature review}

From their survey results of construction industry in US, Ashuri et al. (2014) indicated that remote coordination and regular coordination are the top two most common approaches of conducting coordination, where in remote coordination the team members remotely attend coordination meetings, and in regular coordination the team members conduct regular coordination meetings.

Having projects of UK as examples of applying BIM, Akponeware and Adamu, (2017) stated that all public sector projects in UK were required starting from April 2016, to comply with Level 2 BIM specifications. They maintained that the benefits of specific requirements, however, are still unclear, and argued that what is obvious is that the transformation process is still replete with obsctles including inter-system and intra-system clashes (Akponeware and Adamu, 2017).

Froese et al. (2007) conducted an industrial survey on the Canadian construction IT industry, and concluded to identify the top trends in information technology over the next 10 years that will be important for the construction industry. The trend of using "Web-based collaboration and project management systems" had the major responses (Froese et al., 2007).

Chiu and Lai (2016) described the use of BIM in the Hong Kong construction industry as it is still limited, especially in building services engineering, BSE which covers various disciplines such as electrical, airconditioning, fire services, and plumbing and drainage.

Shen et al. (2010) proposed the integration of 4D or 5D, which integrates time and cost models in addition to the $3 \mathrm{D}$ geometry models, as a method of change management issues. Changes in the projects in this method can be controlled not only in the design and engineering stages but also to some extent in the built environment lifecycle (Shen et al., 2010).

From their study, Wan and Kumaraswamy (2012) stated that "poor coordination among different trades and processes", and "frequent design changes and/or errors" are viewed as two of the major causes of production shortcomings in the pre-installation stage. Many researchers proposed the coordination during the design stage is more effective process than track the clashes in later stages (Chiu and Lai, 2016; Wan and Kumaraswamy, 2012; Malatras et al., 2008).

\subsection{Cases from regional construction projects}

BIM became an essential tool for the projects during designing and construction for its major benefits, such as: coordinating the MEP systems in single multidisciplinary model, reducing waste materials, saving budget and time, helping in project management and making bill of quantities, and many other beneficial tasks. A mediumscale construction firm in the Arabian Gulf, had started applying the BIM method in their construction projects. Based on this construction-firm experience and its design team opinions, reducing the waste materials and coordinating the MEP systems are the most beneficial uses of BIM in their projects. The Head of the design team stated that avoiding the clashes in the design stages is more effective in terms of saving time and effort than conducting the coordination meetings in later stages. The followings are cases of clashes in their construction projects appearing in 3D digital modelling and working drawings details.

The research scope does not cover the types each BSS, for example there are different methods of HVAC that are applied into different systems and types. Without venturing into the details and the components of BSS as well as the various specifications of each BSS used in these real projects, the following example-cases in Fig. 8 and Fig 9 show two different clash cases. In Fig. 8, the clash appears between a HVAC duct and a structural drop panel in 
one position, and two HVAC ducts and a suspended ceiling in another position. In Fig. 9, of the four clashes highlighted is fire system pipes and a HVAC duct.

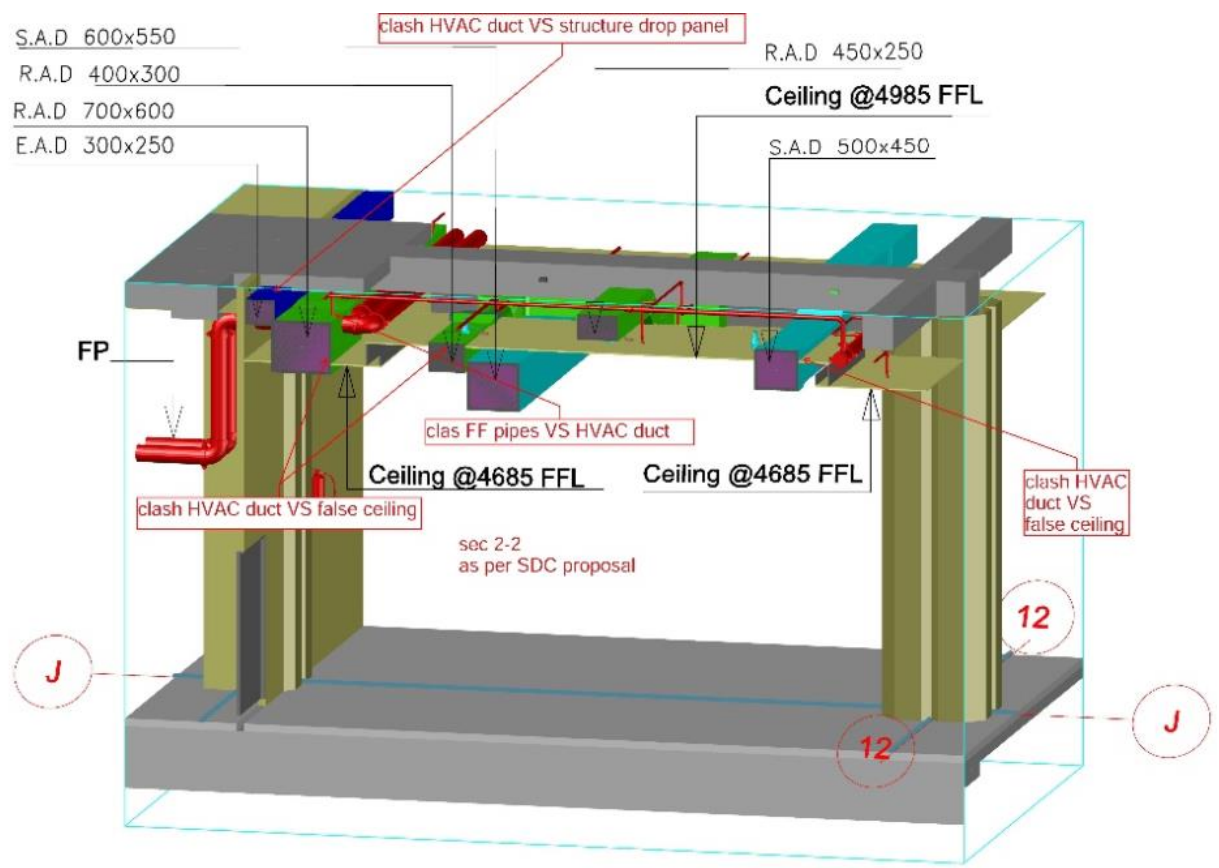

FIG. 8: Service system, HVAC clashes with a structural element, drop panel.

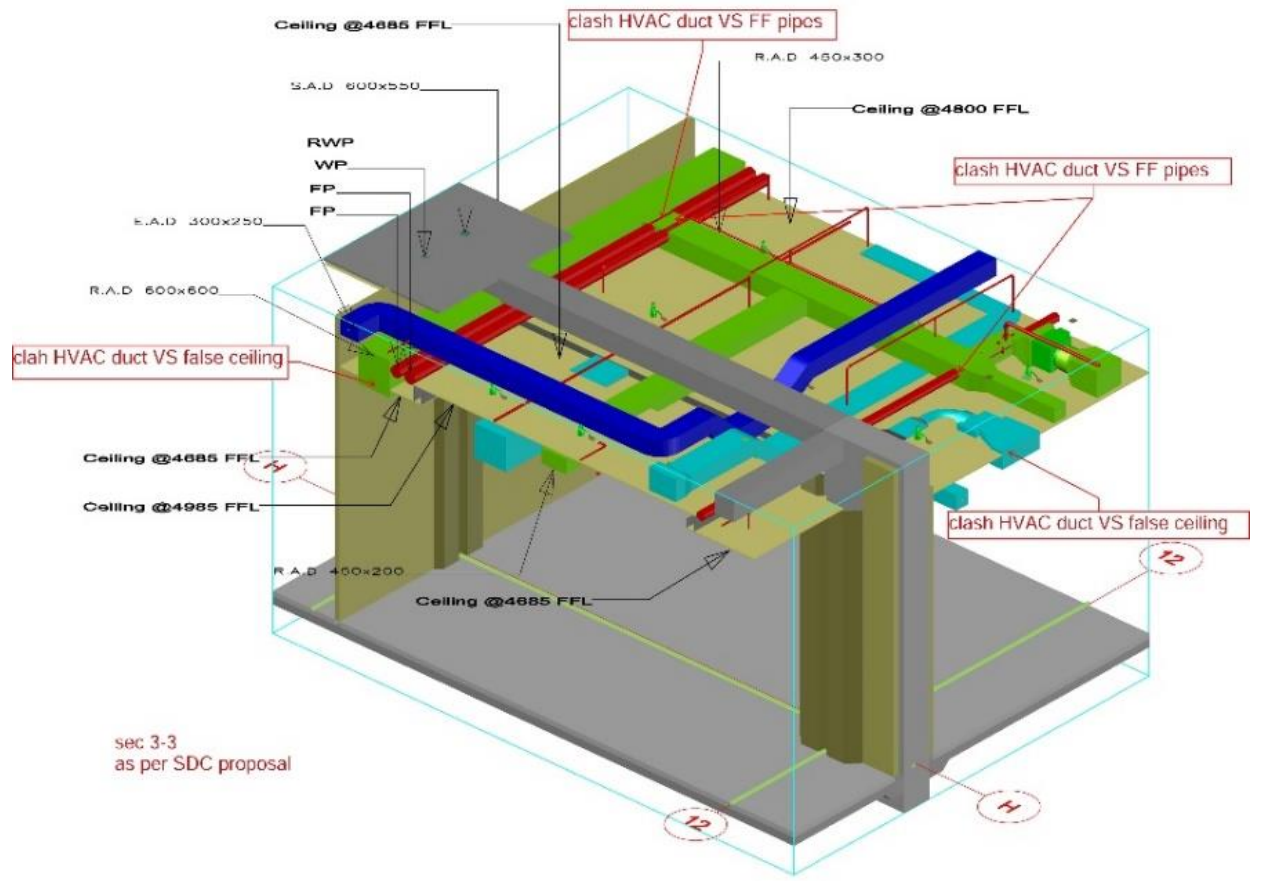

FIG. 9: Service system, HVAC clashes with both fire system pipes and the suspended ceiling.

Fig. 10 shows another clash case in which clashes occur between different systems' pipes network: drain pipe, chilled water pipe, fire pipes, cable tray, and HVAC duct. 


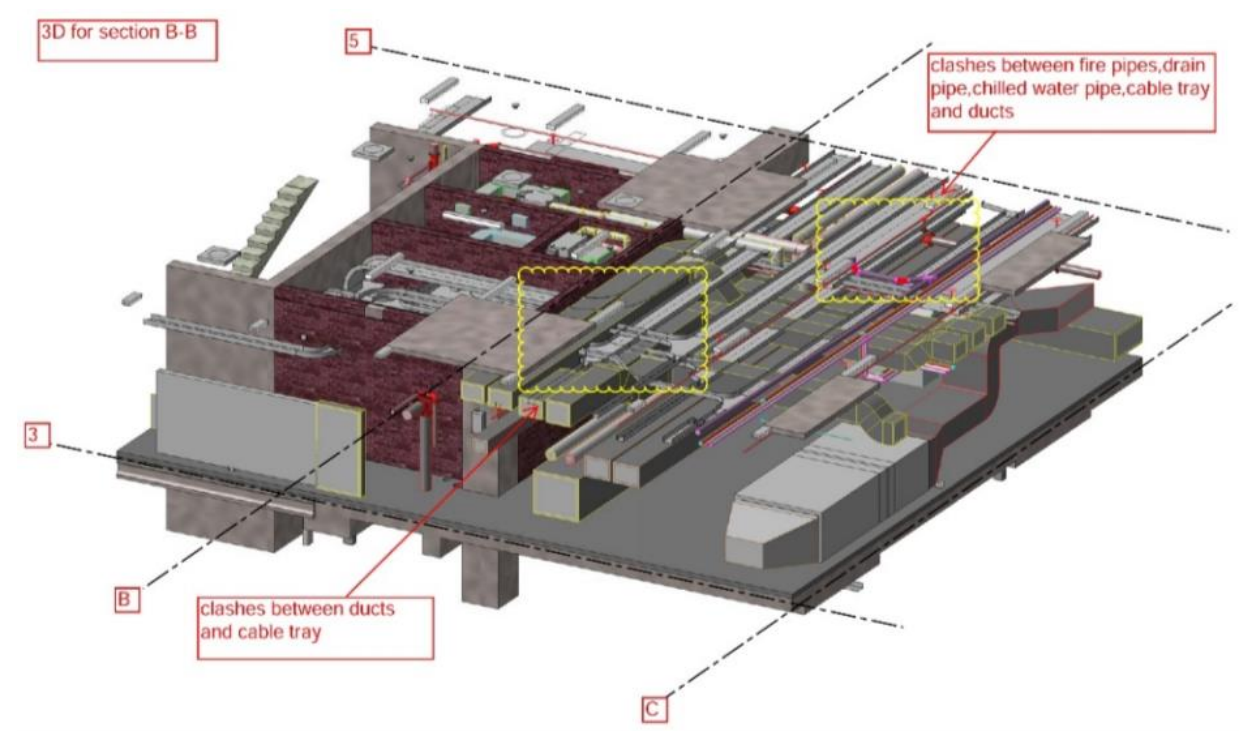

FIG. 10: Clashes between different systems' pipe network: drain pipe, chilled water pipe, cable tray, and HVAC duct.

Fig. 11 shows another clash case in which clashes occur between a drain pipe and a cable tray in one position, a HVAC duct and a room door that is a building component in a second position, and a cable tray and a HVAC duct in a third position.

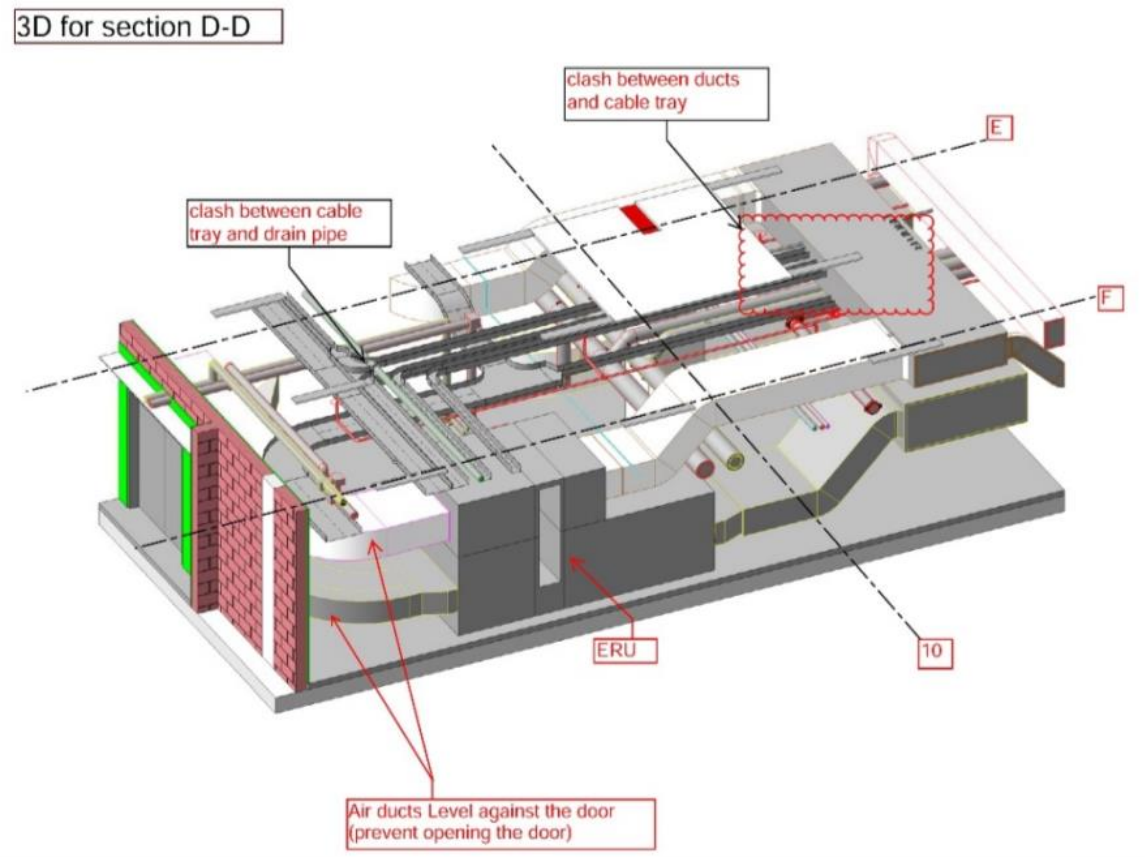

FIG. 11: Service system, HVAC clashes with an architectural element, room door.

\section{CONCLUSIONS}

Due to cultural practices and lack of technologies to support clash avoidance, the clash detection has been favoured over the clash avoidance (Akponeware and Adamu, 2017; Leite, 2011; Benning, 2010). From empirical evidence of their study, Akponeware and Adamu (2017) strongly linked MEP-related clashes to: a) the cultural practices of isolated working among designers, and b) lack of specialised professional training among designers. However, affecting the design process and its current practices, clashes of MEP systems demonstrate the need for certain modifications and changes on how not only designers work but also architecture students learn. 
The paper based on the qualitative analysis of both the real projects of construction industry and the student projects of academia, concludes that integrating the MEP systems into the conceptual design phases eliminates the clashes and conflicts that may occur in later stages, and concurrently the possibility of not detecting these conflicts till the construction process.

The BSS complexity in the students' projects is less than in the practical cases, particularly in including all BSS elements, as well as in defining and modelling more details and components. In order to bridge this gab, training and experience should be provided into the academic level for not only the architecture students but also the students of other specialisations, to gain and accumulate the required BSS knowledge. Theoretical courses to address this technical knowledge are not adequate. Therefore, architecture education professionals have to come up with solutions to include: a) practical training in certain specialisations beside architectural design, site supervision, project management and others, b) lectures by professionals focusing on technical knowledge, and c) regular visits of different project sites to address the technical details and knowledges.

Advantages of using BIM in conducting coordination in the initial design stages have been highlighted in the regional construction firm's project cases for designers, stakeholders and decision makers, in many areas such as BSS coordination and waste-materials saving.

Globally, there are other factors that may cause the MEP clashes due to the current practices, such as: cost issues, project participants related issues, common mistakes and errors produced, project time issues, lack of training and experience, and lack of legal standards or specification to adapt BIM that is a major factor to avoid or expose clashes.

\section{REFERENCES}

Abdelhameed, W. (2018) BIM from Conceptual Model to Construction, 2018 International Conference on Innovation and Intelligence for Informatics, Computing, and Technologies (3ICT), Sakhir, Bahrain, 2018, pp. 1-4. doi: 10.1109/3ICT.2018.8855762.

Abdelhameed, W. and Saputra W. (2019) Smart Solutions and Architectural Design: a framework for building service systems' design. The 2nd Smart Cities Symposium (SCS 2019), University of Bahrain, Sakhir, Bahrain. doi: 10.1049/cp.2019.0196.

Akponeware, A.O. and Adamu, Z.A., (2017). Clash detection or clash avoidance? An investigation into coordination problems in 3D BIM. Buildings, 7(3), p.75.

Arugam, K., Ghadimi, A., and Chang, L.H., (2018). Design and Optimisation of Home Scale Greywater Recycling Package. In MATEC Web of Conferences, Vol. 152, p. 02005. EDP Sciences.

Ashuri, B., Yarmohammadi, S. and Shahandashti, M., (2014). A critical review of methods used to determine productivity of mechanical, electrical, and plumbing systems coordination. In Construction Research Congress 2014: Construction in a Global Network, pp. 777-786.

Benning, P., Dumoulin, C., Dehlin, S., Tulke, J., Åberg, P., Fristedt, S., Holopainen, R., (2010). Report-Collaboration Processes. In Framework for Collaboration. InPro Consortium: Brussels, Belgium, pp. 100-125.

Chiu, B.W. and Lai, J.H., (2016). Implementing Building Information Modelling in Building Services Engineering: Benefits and Barriers. Building up business operations and their logic Shaping materials and technologies, 3, p.332.

Cao, P., Fan, Z., Gao, R. and Tang, J., (2016), August. A framework of a fast any-angle path finding algorithm on visibility graphs based on $\mathrm{A} *$ for plumbing design. In Flexible Automation (ISFA), International Symposium on Flexible Automation, ISFA. IEEE, pp. 333-339.

Dantas Filho, J.B.P., Angelim, B.M., Guedes, J.P., Augusto, M., de Castro, F. and Neto, J.D.P.B., (2015), December. Virtual design and construction of plumbing systems. In International Conference on Engineering, Vol. 2015, pp. 2-4.

Deniz, G.O., (2018). Emerging CAD and BIM trends in the AEC education: an analysis from students' perspective. Journal of Information Technology in Construction, ITcon, 23(7), pp.138-156.

Farooq, J., Sharma, P. and Kumar, S., (2017). Applications of Building Information Modeling in Electrical Systems Design. Journal of Engineering Science \& Technology Review, 10(6).

Froese, T., Han, Z. and Alldritt, M., (2007). Study of information technology development for the Canadian construction industry. Canadian Journal of Civil Engineering, 34(7), pp.817-829. 
Guangbin, W., Wei, L. and Xuru, D., (2011). Exploring the high-efficiency clash detection between architecture and structure. In International Conference on Information Management and Engineering, Singapore.

Gregorius, A. and Widjarnarso, T.H., (2015). BIM Course Development and Its Future Integration at University of Indonesia and Institute of Technology Bandung, Indonesia. In Proceedings of 9th BIM Academic Symposium and Job Task Analysis Review, Washington, DC, pp.10-17.

Hassanain, M.A., Adewale, B., Al-Hammad, A.M. and Sanni-Anibire, M.O., (2018). Factors affecting building services' coordination during the design development and review stages. Built Environment Project and Asset Management, 8(1), pp.64-77.

Korman, T. M., Simonian, L. and Speidel, E. (2010). "How building information modelling has changed the MEP coordination process.” In Ghafoori (Ed.) Challenges, Opportunities and Solutions in Structural Engineering and Construction, Taylor \& Francis, London, 959-63.

Korman, T. M., Fischer, M. A., and Tatum, C.B. (2003). "Knowledge and reasoning for MEP coordination." Journal of Construction Engineering and Management, 129 (6), 627-34.

Leite, F., Akcamete, A., Akinci, B., Atasoy, G., Kiziltas, S., (2011). Analysis of modelling effort and impact of different levels of detail in building information models. Automation Construction, 20, pp. 601-609.

Malatras, A., Asgari, A.H., Baugé, T., and Irons, M., (2008). A service-oriented architecture for building services integration, Journal of Facilities management, vol. 6, pp. 132-151, 2008.

Matsubayashi, M., and Watanabe, S., (2015). Generating Schematic Diagrams of MEP Systems from 3D Building Information Models for Use in Conservation. Emerging Experience in Past, Present and Future of Digital Architecture, Proceedings of the 20th International Conference of the Association for Computer Aided Architectural Design Research in Asia, CAADRIA. Daegu, 20-22 May, pp.293-302.

Othman, A.A.E., (2011). Improving building performance through integrating constructability in the design process. Organization, technology \& management in construction: an international journal, 3(2), pp.333-347.

Olofsson, T., Lee, G., and Eastman, C., (2008). Editorial - Case studies of BIM in use, ITcon, Vol. 13, pp. 244-245.

Palomera-Arias, R. and Liu, R., (2015). Developing BIM laboratory exercises for a MEP systems course in a construction science and management program. In Proceedings of 9th BIM Academic Symposium and Job Task Analysis Review, Washington, DC pp. 88-95.

Pérez-Lombard, L., Ortiz, J., Coronel, J.F. and Maestre, I.R., (2011). A review of HVAC systems requirements in building energy regulations. Energy and Buildings, 43(2-3), pp.255-268.

Shen, W., Hao, Q., Mak, H., Neelamkavil, J., Xie, H., Dickinson, J., Thomas, R., Pardasani, A. and Xue, H., (2010). Systems integration and collaboration in architecture, engineering, construction, and facilities management: A review. Advanced engineering informatics, 24(2), pp.196-207.

Staub-French, S. and Khanzode, A. (2007). "3D and 4D modelling for design and construction coordination, Issues and lessons learned." ITcon, 12, 381-407.

Stone, T., and King, M. (2015). Design Disassembled: Understanding Building Systems through BIM. In Proceedings of 9th BIM Academic Symposium and Job Task Analysis Review, Washington, DC, pp.175-182.

Turk Z. (1991). Integration of Existing Programs Using Frames, CIB Seminar Computer Integrated Future, 16-17 September, Eindhoven, Netherlands.

van den Helm, P., Böhms, M. and van Berlo, L., (2010), June. IFC-based clash detection for the open-source BIMserver. In Computing in civil and building engineering, proceedings of the international conference. Nottingham University Press, Nottingham, UK, Vol. 181.

Wan, S.K.M. and Kumaraswamy, M.M., (2012). Improving building services coordination at the pre-installation stage. Engineering, Construction and Architectural Management, (19), 3.

Wang, J., Wang, X., Shou, W., Chong, H.Y. and Guo, J., (2016). Building information modeling-based integration of MEP layout designs and constructability. Automation in Construction, 61, pp.134-146.

Wang, L., and Leite, F., (2016). Formalized knowledge representation for spatial conflict coordination of mechanical, electrical and plumbing (MEP) systems in new building projects. Automation in Construction, 64, pp.20-26.

Yung, P., Wang, J., Wang, X. and Jin, M., (2014). A BIM-enabled MEP coordination process for use in China. Journal of Information Technology in Construction, ITcon, 19(23), pp.383-398. 